\title{
Numerical simulation on flexural crack induced debonding failure in FRP strengthened concrete beams
}

\author{
Binyu Zheng ${ }^{\mathrm{a}}$, Jianhe Xie and Gongfa Chen \\ School of Civil and Transportation Engineering, Guangdong University of Technology, Guangdong, Guangzhou \\ 510000, China
}

\begin{abstract}
Intermediate crack debonding failure is one of the most common failure modes in the FRP reinforced concrete structures. In order to simulate the formation of debonding failure accurately, this paper adopts an elastic-plastic damage constitutive law to model concrete and take into account the interface of three directions simultaneously, namely the normal direction and the two shear direction of the interface to establish a precise mixture of bilinear cohesive model. The model is used for finite element analysis of debonding failure during loading process in the FRP-concrete interface. Comparisons with experimental results of CFRP concrete joints, the finite element model in this paper can well simulate the damage evolution and debonding process of the FRP reinforced concrete beam in the mid span, which provides a theoretical basis for its wider applications in practical engineering.
\end{abstract}

Keywords: fiber reinforced polymer (FRP), concrete, interface debonding, FEM, elasticplastic model.

\section{Introduction}

As the Fiber Reinforced composites (Fiber Reinforced Polymers, FRP) is light and thin, and has high strength and corrosion resistance and other advantages which overcome the disadvantages of steel reinforced concrete structures, externally bonded FRP sheet reinforcement technology in concrete structures has grown very rapidly in recent years. Due to the brittleness of FRP materials compared with the ductility of steel, the brittleness will limit the ductility of the reinforced concrete beam and the stress redistribution. Therefore more failure modes, exits with the use of FRP.

Many scholars have studied thoroughly for flexural strength of FRP reinforced concrete beams. Lots of experimental results show that major failure modes of reinforced concrete beams with FRP are: 1. FRP pulled off; 2. Concrete compressive zone crushed; 3. Tensile steel pulled off; 4. Shear failure of concrete near the bearing; 5. Debonding failure. The first four forms of failure can be avoided by bonding enough FRP in the bottom and lateral sides of the beam. The fifth debonding failure is a very common form. According to Teng et al.[2003], the debonding failure can be divided into the following four forms:(1) debonding of the protective layer; (2) interfacial debonding of the FRP end; (3) Intermediate crack debonding, called IC debonding in short; (4) debonding due to key inclined cracks.

${ }^{a}$ Corresponding author : 903585753@qq.com 
The major reason for debonding of the protective layer, and FRP end, is FRP truncation causing discontinuity of bending stiffness and stress concentration. Such failure modes can generally be avoided by applying a U-shaped hoop (Garden and Hollaway ,1998) at the end of the FRP. Debonding failure due to key inclined cracks is mainly induced insufficient shear bearing capacity of the beam, so that inclined cracks occurs which leads to the member's ultimate failure of the beam. Such damage can be avoided by utilizing sufficient shear reinforcement. IC debonding failure is due to too large bending crack, which leads to local bond stress centralized between the cracks near the FRP sheets and concrete. Such debonding failure in FRP reinforced concrete beams are common and lots of researches mainly resorts experimental tests. In respect to the finite element analysis, many scholars have conducted simulations of IC debonding (Wu and Niu 2000, Wong and Vecchio 2003, Wu and Yin 2003). In these analyses, most consider one or two directions of the interface bonding effect in the 3D model. Wang (2006) used a cohesive zone model based on nonlinear fracture mechanics to simulate FRP-concrete interface bonding effect. Teng and Lu (2007) proposed a dual peeling failure criterion that can better simulate the behavior of peeled FRP-concrete interface. Moreover, the model is only applicable for the fine unit $(0.25 \mathrm{~mm} \sim 0.5 \mathrm{~mm})$. For ordinary concrete unit, a self-developed interface element is necessary to simulate the interface bonding effect and the shear transfer coefficients are generally difficult to be determined.

In summary, a thorough research is necessary for such failure of the CFRP reinforced concretesteel beam. Based on the study of Camanho (2002) and Teng (2015), a precisely mixed-mode bilinear cohesive zone model is established which accounts the normal and shear directions of the interface . The proposed model is verified properly by comparing the experimental results.

\section{Finite element model}

In this paper, an elastic-plastic damage constitutive law is used for concrete constitutive while an ideal elastic-plastic relationship is used for rebar. In this article, CFRP is treated as an ideal linear elastic material. A cohesive zone model based on fracture mechanics is used in this paper to simulate the debonding failure of the interface between CFRP and concrete beam. The test specimen and the finite element model are shown in Figures 1 and 2 respectively.

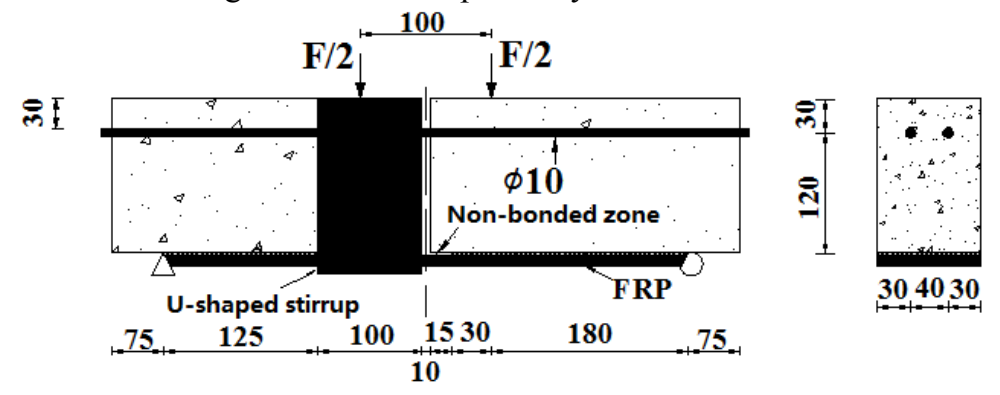

Figure 1. The specimen size.

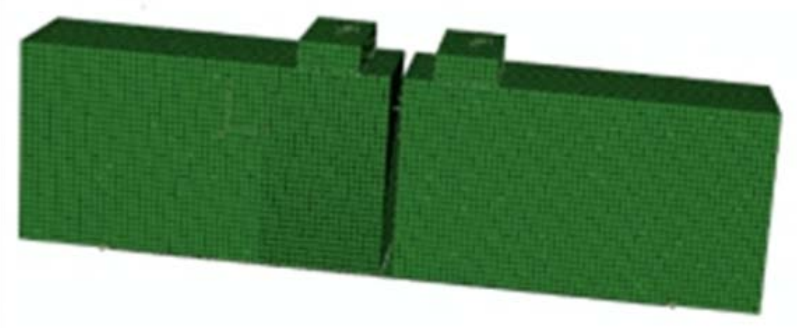

Figure 2. Finite element model. 


\subsection{Cohesive zone model}

Using FRP to reinforce concretes structure can effectively improve the ultimate load, yield load and stiffness of structures. The bonding performance of the interface between the FRP composite and the concrete structures is a key factor to the performance of the reinforced structures. In ABAQUS, a cohesive cell can be used to simulate the bonding properties of the interface. There are three common cohesive traction-displacement relationships: hyperbolic, bilinear and parabolic. The bilinear model (as shown in Figure 3) is simple, which can be easily used in the finite element analysis and better simulate the behavior of the bonding. The accurate mixed-mode bilinear cohesive model which timely takes interface normal direction and two shear directions into consideration is adopted in this paper, that is the bonding separation in the normal direction of the interface (mode I ) and the bond slip of the two shear direction (mode II ) which is vertical to the normal direction.

The bilinear mixed-mode cohesive model considers the traction-separation relationship in the normal direction and the two shear directions of the interface. In this paper, $t_{n}, t_{s}$ and $t_{t}$ are used for the stress in the normal direction and the two shear directions respectively, while $\delta_{n}, \delta_{s}$ and $\delta_{\text {t }}$ represent cracking displacement in normal direction and the two shear directions respectively as well as T0 is the original thickness of cohesive element.

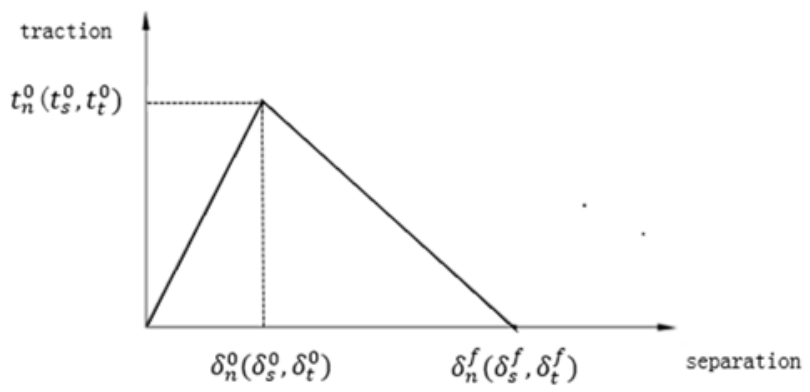

Figure 3. Bilinear mixed-mode cohesive constitute.

\subsubsection{The elastic stage}

Assume that before the damage of bonding layer, CFRP-concrete interface is linear elastic, and the interface traction and cracking displacement can be represented by the following equation (1):

$$
\left\{\begin{array}{l}
t_{n}=k_{n n} \delta_{n} \\
t_{s}=k_{s s} \delta_{s} \\
t_{t}=k_{t t} \delta_{t}
\end{array}\right.
$$

Where $k_{n n}, k_{s s}, k_{t t}$ are the initial stiffness in the interface normal direction and the two shear direction respectively and appropriate values selected according to the references (Camanho and Davila 2002; Teng, Fernando et al., 2015).

\subsubsection{The damage evolution}

Using QUADS to judge the initiation of the interface debonding:

$$
\left(\frac{\left\langle t_{n}\right\rangle}{f_{t}}\right)^{2}+\left(\frac{t_{s}}{\tau_{\max }}\right)^{2}+\left(\frac{t_{t}}{\tau_{\max }}\right)^{2}=1
$$


where $<>$ is Macaulay bracket that indicates initiation of the interface debonding will not occur under the compressive stress, namely when the normal stress is negative in the glue-thickness direction, the $\left\langle t_{n}\right\rangle$ value is zero.

In order to describe the mixed-mode model, which considers the damage evolution in the normal direction and the two shear direction simultaneously, the effective displacement $\delta_{m}$ and damage factor $\mathrm{D}$ are introduced according to Camanho and Davila (2002).

$\mathrm{D}=0$ and $\mathrm{D}=1$ correspond to no damage and complete failure of glue. When glue is in linear softening stage, damage variable $\mathrm{D}$ varies from 0 to 1 . Several damage evolution criteria are defined for the cohesive model under the mixed crack fracture mode, including Power Law and B-K law (Benzeggagh and Kenane 1996). B-K law is adopted in this paper:

$$
G_{n}^{C}+\left(G_{s}^{C}-G_{t}^{C}\right)\left(\frac{G_{S}}{G_{T}}\right)^{2}=G^{C}
$$

where $G_{s}=G_{s}+G_{t}, G_{T}=G_{n}+G_{s}, G_{s}^{C}=G_{t}^{C} ; G_{n}, G_{s}$ and $G_{t}$ represent respectively the fracture energy of the interface in the normal direction and the two shear direction when the interface is in the complete debonding failure. $G_{n}^{C}$ is the fracture energy of mode I, $G_{s}^{C}$ and $G_{t}^{C}$ are mode II. While $\alpha$ is a material parameter, when takes a value of 1 according to the literature [10], then B - K law becomes the linear fracture criterion.

\section{Results of finite element analysis}

\subsection{Debonding comparisons between FEA and experiment}

The object of this paper is CFRP-concrete specimens, herein called C40 group. In the finite element analysis, CFRP concrete beam began debonding around the mid-span in the non-reinforced end, then it gradually extends to the free end. This is consistent with the experimental observation, as shown in Figures 4a,b,c and Figure5. No debonding happens to the reinforcement end, which was not shown in Figure 4.

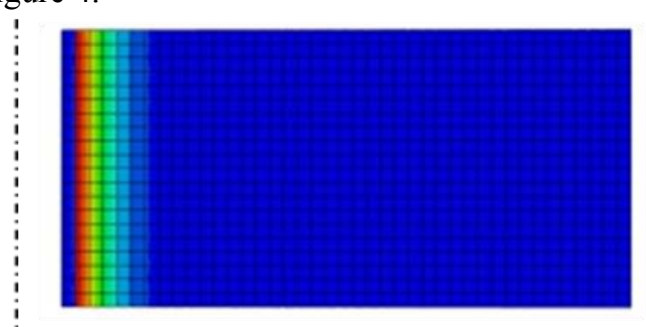

midspan a. pre-debonding

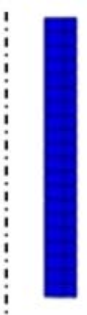

midspan

c. ultimate debonding

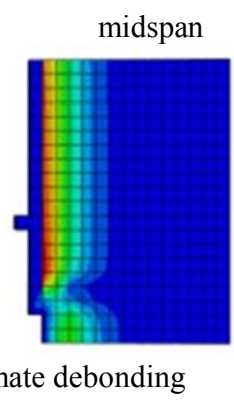

Figure 4. Interface debonding predicted by FEM. 


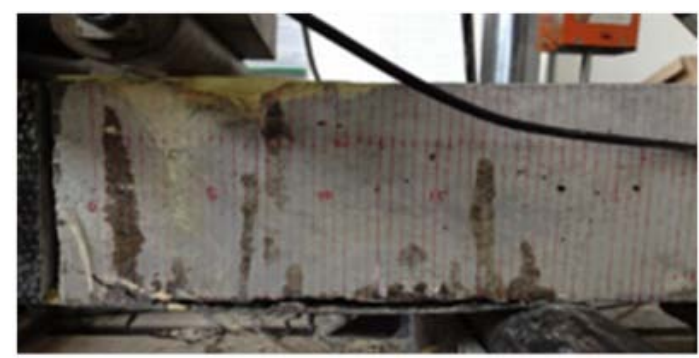

Figure 5. Debonding observed in experimental test.

\subsection{Load - displacement curve comparison}

The figure 6 shows the load-displacement curves of the CFRP-concrete beam obtained by the finite element analysis and the experimental tests, in which the horizontal axis represents the mid-span displacement and the vertical axis represents the load on the specimen. As shown in the diagram, the finite element results are consistent with those of the experimental tests in the load-displacement curves. The experimental ultimate load is $32.0 \mathrm{KN}$ while the finite element calculation is $30.4 \mathrm{KN}$, which means that the relative error is $5.2 \%$.

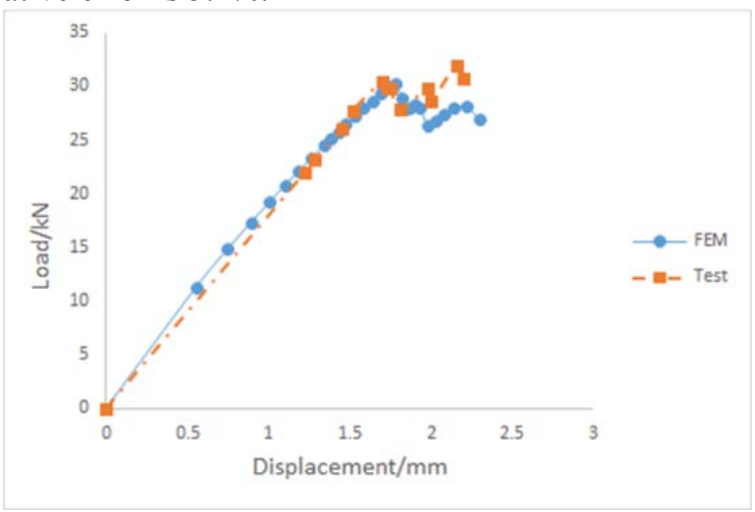

Figure 6. Load-deflection curves.

\subsection{CFRP strain distribution}

Figures 7-9 respectively represents the comparisons of the CFRP strain distribution for the stage of pre-debonding (the FE load is $11.36 \mathrm{KN}$, the test load $10 \mathrm{KN}$ ), initial debonding (the FE load is 27.41 $\mathrm{KN}$, the test load $25 \mathrm{KN}$ ) and complete debonding (the FE load is $25.92 \mathrm{KN}$, the test load $30 \mathrm{kN}$ ). As shown in the figure, the CFRP strain distribution of the finite element calculation is generally consistent with the experimental results. The strain of CFRP reaches the maximum in the middle span, the farther away from the mid-span, the smaller the CFRP strain is, and CFRP strain approaches to 0 at the fixed end. With the increasement of load, CFRP strain increases rapidly, that is, CFRP is subjected to a larger force. 


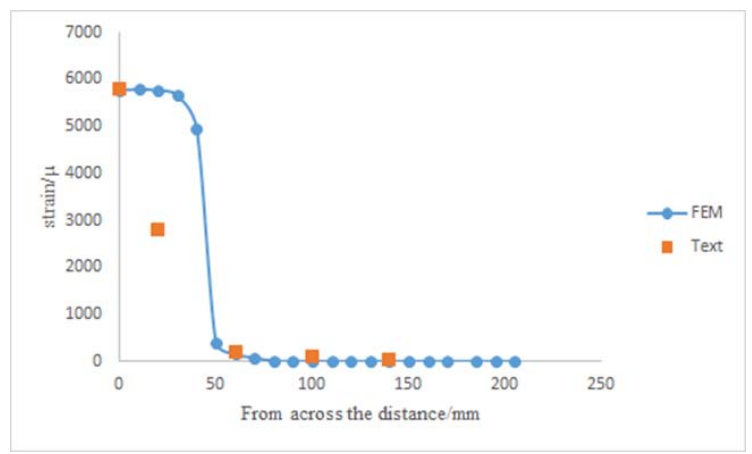

Figurg 7. CFRP strain curves before debonding.

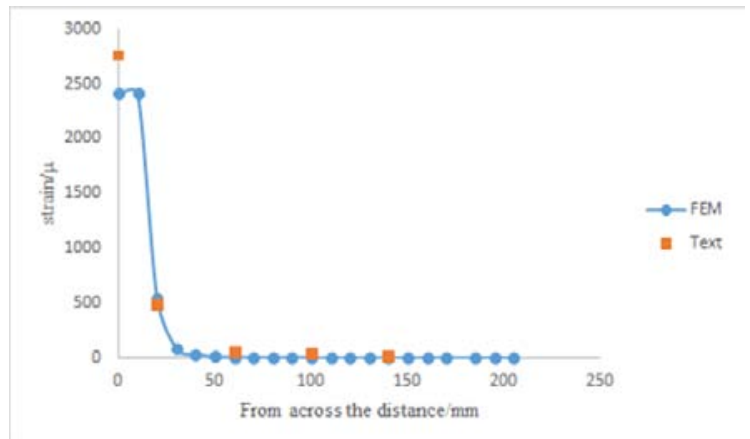

Figure 8. CFRP strain curves at the onset of debonding.

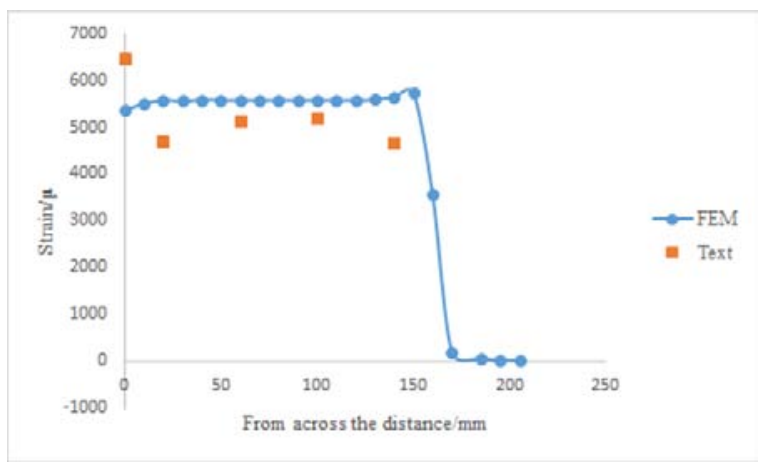

Figure 9. CFRP strain curves at the ultimate debonding.

\section{Conclusions}

(1) The results obtained by the finite element analyses are in good agreement with the experimental ones. In the initial stage, the load and the deflection increase linearly, while the load decreases slightly after reaching the peek load with the increase of the deflection.

(2) The interface debonding predicted by the finite element analyses evolves from the middle of the span to the fixed end, which is consistent with the experimental observations.

(3) The CFRP strain in the span center predicted by the finite element analyses is the largest, the closer to the fix end, the smaller the strain is; with the increase of the load, the CFRP strain increases. But in Figure 9, at the distance of $20 \mathrm{~mm}$ away from the span center the CFRP strain is $5767 \mu$ by the finite element analysis, the CFRP strain observed in the experimental test is $2792 \mu$. As the test 
specimen is not symmetrically supported, to a certain extent, it led to the error between the finite element calculation and the results of the experiment. However, because strain gauge is not measured from the point of $160 \mathrm{~mm}$ away from span center to bearing end (in Figure 9), the test curve is lack of the descending segment. Therefore, the distribution of CFRP strain from the span center to $160 \mathrm{~mm}$ from the span center is consistent with the experimental results.

In summary, the accurate bilinear mixed-mode cohesive model which considers the three interface directions can predict the mid-span debonding failure process of CFRP reinforced concrete structures. It has advantages compared with experimental tests which request a number of specimens, a long test cycle and other shortcomings. The paper provides the theoretical basis for the application of the finite element analyses in practical reinforced engineering.

\section{References}

1. Teng, J. G., S. T. Smith, J. Yao and J. F. Chen (2003). "Intermediate crack-induced debonding in RC beams and slabs." Construction \& Building Materials 17(6-7): 447-462.

2. Garden, H. N. and L. C. Hollaway (1998). "An experimental study of the influence of plate end anchorage of carbon fibre composite plates used to strengthen reinforced concrete beams." Composite Structures 42(2): 175-188.

3. Wu, Z. S. and H. D. Niu (2000). "Study on debonding failure load of RC beam strengthened with FRP sheets." Journal of Structural Engineering A.

4. Wong, R. S. Y. and F. J. Vecchio (2003). "Towards modeling of reinforced concrete members with externally bonded fiber-reinforced polymer composites." Aci Structural Journal 100(1): 4755 .

5. Wu, Z. and J. Yin (2003). "Fracture behaviors of FRP-strengthened concrete structures." Engineering Fracture Mechanics 70(10): 1339-1355.

6. Wang, J. (2006). "Cohesive zone model of intermediate crack-induced debonding of FRP-plated concrete beam." International Journal of Solids \& Structures 43(21): 6630-6648.

7. Lu, X. Z., J. G. Teng, L. P. Ye and J. J. Jiang (2007). "Intermediate Crack Debonding in FRPStrengthened RC Beams: FE Analysis and Strength Model." Journal of Composites for Construction 11(2): 161-174.

8. Camanho, P. P. and C. G. Davila (2002). "Mixed-Mode Decohesion Finite Elements for the Simulation of Delamination in Composite Materials. NASA/TM-2002-211737."

9. Teng, J. G., D. Fernando and T. Yu (2015). "Finite element modelling of debonding failures in steel beams flexurally strengthened with CFRP laminates." Engineering Structures 86: 213-224.

10. Benzeggagh, M. L. and M. Kenane (1996). "Measurement of mixed-mode delamination fracture toughness of unidirectional glass/epoxy composites with mixed-mode bending apparatus." Composites Science \& Technology 56(4): 439-449. 